\title{
Molecular genetic analyses of the TMPRSS2-ERG and TMPRSS2-ETV1 gene fusions in 50 cases of prostate cancer
}

\author{
MARTA WINNES $^{1}$, ERIK LISSBRANT $^{2}$, JAN-ERIK DAMBER $^{2}$ and GÖRAN STENMAN ${ }^{1}$ \\ ${ }^{1}$ Lundberg Laboratory for Cancer Research, Department of Pathology; ${ }^{2}$ Department of Urology, \\ the Sahlgrenska Academy at Göteborg University, Sahlgrenska University Hospital, SE-413 45 Göteborg, Sweden
}

Received November 16, 2006; Accepted December 19, 2006

\begin{abstract}
Recently, gene fusions between the androgen responsive gene TMPRSS 2 and members of the ETS-family of DNA-binding transcription factor genes were found in prostate cancer. Recurrent fusions were identified between the 5'-noncoding region of TMPRSS2 and ERG, or less frequently ETV1 or ETV4, resulting in overexpression of normal or truncated ETS-proteins. Herein, we have analyzed a series of 50 prostate cancer samples for expression of TPRSS2-ERG and TMPRSS2-ETV1 fusion transcripts. RT-PCR analysis revealed TMPRSS2-ERG fusion transcripts in 18 of the 50 tumors $(36 \%)$. None of the tumors expressed a TMPRSS2ETV1 fusion. Our findings show that the TMPRSS2-ERG fusion is common in prostate cancer and that the related TMPRSS2-ETV1 fusion is very rare. However, the frequency of $E R G$-fusions in the present study is somewhat lower than previously observed, indicating heterogeneity with regard to expression of ETS-gene fusions in subsets of prostate cancers. Moreover, clinical follow-up studies showed a clear tendency that fusion-positive tumors were associated with lower Gleason grade and better survival than fusion-negative tumors. Our findings suggest that ERG gene fusions might be of prognostic significance in prostate cancer.
\end{abstract}

\section{Introduction}

In developed countries, prostate cancer is the second most commonly diagnosed cancer and the third most common cause of cancer death among men $(1,2)$. In addition to patient's age and race, a positive family history is probably the strongest known risk factor for prostate cancer (3). In its clinical form, prostate cancer is diagnosed as local or advanced, and treatments range from surveillance, to radical local treatment,

Correspondence to: Professor Göran Stenman, Lundberg Laboratory for Cancer Research, Department of Pathology, Göteborg University, Sahlgrenska University Hospital, SE-413 45 Göteborg, Sweden

E-mail: goran.stenman@1lcr.med.gu.se

Key words: prostate cancer, fusion gene, TMPRSS2, ERG, ETV1, prognosis or androgen deprivation therapy. Androgen deprivation reduces clinical symptoms in $\sim 70-80 \%$ of patients with advanced prostate cancer, but most tumors relapse within two years to an androgen independent state (4), and so far it has not been possible to cure patients with metastatic disease. In order to find new therapeutic pathways, the genetic mechanisms behind prostate cancer initiation and tumor progression towards androgen independence and metastases need to be thoroughly elucidated.

An important breakthrough in the search for novel pathogenetic mechanisms in prostate cancer was recently reported when fusion oncogenes involving members of the ETS-family of transcription factor genes were identified in prostate cancer $(5,6)$. Recurrent fusions were found between the 5'-noncoding region of the TMPRSS 2 gene and the ERG, ETV1 or ETV4 genes, resulting in overexpression of normal or truncated ETS-proteins. The fusions were found in $45-79 \%$ of prostate cancer samples. The TMPRSS2-ERG fusion is by far the most common fusion type followed by TMPRSS2-ETV1 and TMPRSS2-ETV4, in that order of frequency.

The ERG, ETV1 and ETV4 genes all encode transcription factors containing a highly conserved, sequence-specific DNAbinding domain, the so-called ets domain (7). For reasons that remain unclear ETS-family genes are frequently targeted by chromosomal rearrangements in various human malignancies, resulting in overexpression of oncogenic ETS-gene fusions (7). The TMPRSS 2 gene encodes an androgen regulated, type II transmembrane-bound serine protease that is highly expressed in normal prostate tissue as well as in neoplastic prostate epithelium $(8,9)$. However, the in vivo biological activities of TMPRSS2 are unclear and recent studies have shown that mice lacking the Tmprss2-encoded protease are viable and fertile and have no detectable abnormalities, suggesting functional redundancy involving one or more of the type II transmembrane serine proteases or other serine proteases (10). The molecular consequences of the ETS-gene fusions in prostate cancer are unclear. In vitro experiments have suggested that the ectopic expression of ETS-family members is mediated by androgen-responsive elements in the TMPRSS 2 promoter (5).

To obtain additional information about the incidence and potential clinical significance of ETS gene fusions in prostate cancer we have screened a series of 50 prostate cancer specimens for expression of the TMPRSS2-ERG and TMPRSS2-ETV1 gene fusions. RT-PCR analysis revealed 
TMPRSS2-ERG fusion transcripts in $36 \%(18 / 50)$ of the tumors. None of the tumors expressed the TMPRSS2-ETV1 fusion. Clinical follow-up studies showed a clear tendency that fusion-positive tumors were associated with lower Gleason grade and better survival compared with fusion-negative tumors.

\section{Materials and methods}

Patients. Prostate tumor biopsis were obtained from 50 patients presenting with clearly palpable tumors of the prostate at the outpatient Urology Clinic at Sahlgrenska University Hospital in Gothenburg, Sweden from April 2000 to February 2003. The median age of the patients was 76 years (range 53.7-86.2 years). Local tumor stage was determined by digital rectal examination. A transrectal ultrasound (TRUS) investigation was subsequently performed and 2-5 ultrasound-guided diagnostic needle biopsies were taken from the tumor under the protection of per oral antibiotic prophylaxis. Biopsies were fixed in formaldehyde according to routine procedures. At the same time, two additional TRUS-guided biopsies were taken from the same part of the tumor as the diagnostic biopsies. These biopsies were instantly frozen in liquid nitrogen and stored at $-70^{\circ} \mathrm{C}$. All patients had histologically confirmed prostatic carcinomas with a Gleason score ranging from $3+3=6$ to $5+5=10$. The presence of cancer in the frozen biopsies was not confirmed histologically, but the probability of them containing cancer was very high since in 39 patients, cancer was found in all diagnostic biopsies and in the 11 remaining patients, cancer was found in more than half of the biopsies. There were 16 T2, 28 T3, and 6 T4 tumors. M-stage was determined by radionuclide scanning and signs of distant metastases (M1) were found in 26 cases. A negative radionuclide scanning (M0) was found in 12 cases and in 12 cases no scanning was performed (Mx). All patients received endocrine treatment either by subcutaneous injection of a $\mathrm{GnRH}$ analog once every 3 months or by surgical castration. A pretreatment PSA level was established prior to the first visit with the urologist and following the onset of treatment, PSA was monitored regularly every three months. In April 2006, 31 patients $(62 \%)$ had died. The cause of death for these patients was determined by examination of death certificates. Ethics approval for use of these samples was obtained from the Ethics Committee of the Sahlgrenska University Hospital, Göteborg, Sweden (No. 667-05).

Statistical analyses. Kaplan-Meier analyses were performed to evaluate progression-free survival and cause-specific survival. The $\chi^{2}$-test was used to evaluate possible differences between fusion-positive and fusion-negative patients with regard to Gleason score and time to PSA progression.

Isolation of RNA, RT-PCR, and nucleotide sequence analyses. Total RNA was extracted from frozen tumor biopsies using the RNeasy micro kit (Qiagen). For cDNA synthesis, $1 \mu \mathrm{g}$ of total RNA was reverse-transcribed using the Superscript ${ }^{\mathrm{TM}}$ First-strand synthesis system according to the manufacturer's manual (Invitrogen). As a control for intact RNA and cDNA, RT-PCR reactions for expression of the housekeeping gene GAPDH (glyceraldehyde-3-phosphate dehydrogenase) was

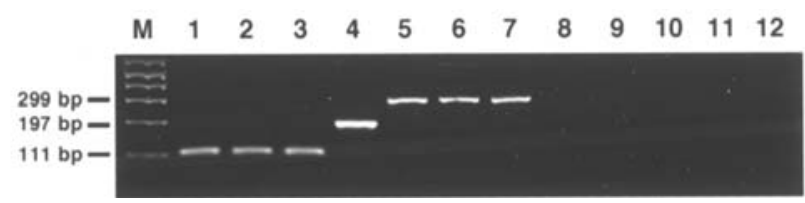

Figure 1. Expression of TMPRSS2-ERG fusion transcripts in prostate cancer. RT-PCR analysis using the primers TMPRSS2-301F and ERG-304R revealed three types of chimeric transcripts, consisting of TMPRSS 2 exon 1 fused to either $E R G$ exon 4 (lanes 1-3), ERG exon 3 (lane 4) or $E R G$ exon 2 (lanes 5-7). Lanes 8-11 represent four fusion-negative prostate tumors. The control lane (lane 12) represents a PCR reaction with fusion-specific primers, but without cDNA template. Sizes of TMPRSS2-ERG fusion transcripts are indicated.



Figure 2. Fusion oncogenes involving the ETS-family members ERG, ETV1 and ETV4 in human cancer. In all fusions depicted, the TMPRSS2, EWSR1 and FUS genes are disrupted and their 5'-parts fused to the 5'-ends or central parts of ETS-genes. Note that the FUS-ERG fusion has been detected in both Ewing's sarcoma and acute myeloid leukemia, whereas the other fusions appear to be tumor type specific.

performed on all cDNAs using the primers GAPDH-48F 5'-GT GAAGGTCGGAGTCAACG-3' and GAPDH-48R 5'-GGTG AAGACGCCAGTGGACTC-3'.

The TMPRSS2-ERG fusion transcript was amplified with the primers TMPRSS2-301F 5'-CAGGAGGCGGAGGCG GA-3' and ERG-304R 5'-GTAGGCACACTCAAACAACG ACTGG-3' or TMPRSS2-301F 5'-CAGGAGGCGGAGG CGGA-3' and ERG-305R 5'-GGCGTTGTAGCTGGGG GTGAG-3' (5). The TMPRSS2-ETV1 fusion transcript was amplified with the primers TMPRSS2-301F 5'-CAGGAGG CGGAGGCGGA-3' and ETV1-302R 5'-CAGGCCATGAA AAGCCAAACTT-3' or TMPRSS2-301F 5'-CAGGAGGCG GAGGCGGA-3' and ETV1-303R 5'-TTGTGGTGGGAAG GGGATGTTT-3' (5). PCR products were gel purified and sequenced using the ABI PRISM ${ }^{\circledR}$ BigDye $^{\mathrm{TM}}$ terminator cycle sequencing ready reaction v2.0 kit (Applied Biosystems, Foster City, CA) in an ABI Prism 310 genetic analyzer (Applied Biosystems). The resulting sequences were analysed using the bioinformatics tools provided by the National Center for Biotechnology Information (NCBI, http://www.ncbi.nlm. nih.gov).

\section{Results and Discussion}

By using RT-PCR we screened a series of 50 prostate cancer specimens for expression of the TMPRSS2-ERG and TMPRSS2-ETV1 gene fusions. Chimeric transcripts corresponding to TMPRSS2-ERG fusions were detected in 18 of the 50 tumors $(36 \%)$. In contrast, none of the tumors expressed TMPRSS2-ETV1 fusion transcripts. Three types of TMPRSS2-ERG chimeric transcripts were detected, consisting of TMPRSS 2 exon 1 fused to either ERG exon 4 (14 cases), 
Table I. Time to PSA progression, metastases and Gleason score in relation to TMPRSS2-ERG status.

\begin{tabular}{lccc}
\hline & & $\begin{array}{c}\text { TMPRSS2-ERG } \\
\text { positive patients }\end{array}$ & $\begin{array}{r}\text { TMPRSS2-ERG } \\
\text { negative patients }\end{array}$ \\
\hline Time to PSA & $\leq 1$ year & $3 / 14(21 \%)$ & $11 / 14(79 \%)$ \\
progression & $>1$ year & $14 / 34(41 \%)$ & $20 / 34(59 \%)$ \\
Metastases & Yes & $9 / 26(35 \%)$ & $17 / 26(65 \%)$ \\
& No & $5 / 12(42 \%)$ & $7 / 12(58 \%)$ \\
Gleason score & $\leq 3+4$ & $8 / 14(57 \%)$ & $6 / 14(43 \%)$ \\
& $\geq 4+3$ & $10 / 36(28 \%)$ & $26 / 36(72 \%)$ \\
\hline
\end{tabular}

$E R G$ exon 2 (four cases; GenBank accession no. DQ831522) or $E R G$ exon 3 (one case; GenBank accession no. DQ831521) (Fig. 1). TMPRSS2 exon 1 - ERG exon 3 represents a novel fusion transcript. All fusion transcripts were confirmed by using a second combination of PCR primers as well as by nucleotide sequence analysis of the PCR products.

The present investigation confirms and extends previous data showing that the TMPRSS2-ERG fusion is recurrent in prostate cancer $(5,11)$. However, the frequency of $E R G$-fusions in our study is somewhat lower than that recently reported, indicating heterogeneity with regard to expression of ETS-gene fusions in subsets of prostate cancers. Thus, Tomlins et al in their original study (5) found TMPRSS2-ERG fusion transcripts in 19 of $42(45 \%)$ samples of localized (32 samples) and metastatic (10 samples) prostate cancer and a TMPRSS2-ETVI fusion in one sample (2.4\%). In the same study, FISH analysis of an independent tissue microarray containing core biopsies from 13 cases of localized and 16 cases of metastatic prostate cancer revealed evidence of ERG- or ETVI-fusions in 23 of the 29 cases $(79.3 \%)$. A similar frequency of ERG-fusions was recently also reported in a small series of 18 prostate cancer samples (11). As pointed out by Tomlins et al the frequency of ETS-gene fusions in their study might have been overestimated due to the fact that the FISH assay used can also detect other $E R G$ rearrangements (5). Similarly, we cannot exclude the possibility that the frequency of ETS-fusions in our material might be slightly underestimated because of shortage of tumor cells in single biopsies. In the present study, the tumor samples were obtained from patients with more advanced prostate cancer compared with the study reported by Tomlins et al (5) who mainly used specimens from radical prostatectomies usually representing early forms of prostate cancer. In accordance with the hypothesis that the fusion is an early event in prostate cancer development that preferentially occurs in low-grade tumors, the number of fusion-positive tumors was lower in the present set of tumors compared to previous studies. However, the true incidence of the various ETS-gene fusions in prostate cancer is still unknown and has to be determined in large, unselected series of histologically confirmed localized and metastatic prostate cancers.

The reason why $E R G$-fusions are much more common in prostate cancer than other ETS-fusions is unknown. The frequency of variant gene fusions may depend on a number of factors, including the transcriptional orientation and the



Figure 3. Gleason score in relation to fusion gene status in 50 prostate cancers

exon-intron organization of the partner genes as well as the size of the introns in which the breakpoints occur (12). Also, factors such as the normal cell type-specific, higher-order spatial organization of chromatin and genes in interphase cells and the nucleotide sequences at the breakpoint junctions may influence the likelihood by which a certain variant gene fusion is generated $(12,13)$. According to publicly available sequence databases, TMPRSS 2 has the same transcriptional orientation as $E R G$ and is located only $\sim 3$ megabases centromeric to $E R G$ within 21q22.3. A fusion between TMPRSS2 and ERG can thus be generated by a simple, cytogenetically cryptic deletion of a small DNA segment containing the 3'-part of TMPRSS2 and the 5'-part of $E R G$ (14), whereas a fusion between for example TMPRSS2 and ETV1 requires multiple rearrangements due to the transcriptional orientation of the two genes on different chromosomes. In Ewing's sarcoma, which typically are characterized by fusions of the EWSR 1 gene to members of the ETS-gene family (Fig. 2), ERG-fusions are at least 10-times more common than ETV1-fusions (15). Since the 5'-partner genes in the prostate- and Ewing-specific ETS-fusions are different, the preference for $E R G$ or ETVI to participate in a particular gene fusion is likely to be due to inherent functional or structural properties of these genes. Cryptic gene fusions have also been found in several other types of neoplasms, for example in leukemias (16-18), salivary gland mucoepidermoid carcinomas and pleomorphic adenomas $(19,20)$, as well as in sarcomas $(21,22)$. Together with the present results, these and other studies (23) indicate that fusion genes might be much more common in human neoplasms, particularly in epithelial tumors, than previously anticipated.

Recent studies have shown that ERG is overexpressed in the majority of prostate cancers and that patients with high $E R G$ overexpression ( $>100$-fold or 2-100-fold) in their prostate tumors have significantly longer PSA recurrence-free survival compared to those with lower expression levels ( $<2$-fold) (24). This observation prompted us to examine the clinical and histopathological significance of expression of the TMPRSS2-ERG gene fusion in the present set of patients (Table I). There was no statistically significant difference in outcome between fusion-positive and fusion-negative patients with regard to cause-specific survival or progression-free survival (data not shown). The TMPRSS2-ERG fusion gene 
was found in $21 \%$ of patients with a PSA progression-free survival of $\leq 1$ year and in $41 \%$ of patients with a progressionfree survival of $>1$ year (Table I). Moreover, the fusion was observed in $57 \%$ of the tumors with a Gleason score of $(3+4$ and in $28 \%$ of tumors with a Gleason score of $\geq 4+3$ ( $p=0.099$; Table I and Fig. 3). Although there is a clear tendency that fusion-positive tumors are associated with better survival and lower Gleason score than fusion-negative tumors, there were no statistical differences between the groups. This could be related to the fact that the study sample was too small and/or that the fusion bears little or no prognostic information. Additional studies are therefore warranted before firm concusions can be made as to the clinical significance of a molecular classification of prostate cancers into fusion-positive and fusion-negative tumors.

\section{Acknowledgements}

This study was supported by grants from the Swedish Cancer Society and the IngaBritt and Arne Lundberg Research Foundation. We thank Barbro Wedell for excellent technical assistance and Ulric Pedersen for expert help with the illustrations.

\section{References}

1. Parkin DM, Bray F, Ferlay J and Pisani P: Global cancer statistics, 2002. CA Cancer J Clin 55: 74-108, 2005.

2. Boyle P and Ferlay J: Cancer incidence and mortality in Europe, 2004. Ann Oncol 16: 481-488, 2005.

3. Grönberg H, Damber L and Damber J-E: Familial prostate cancer in Sweden. A nationwide register cohort study. Cancer 77: 138-143, 1996.

4. Sogani PC and Fair WR: Treatment of advanced prostatic cancer. Urol Clin North Am 14: 353-371, 1987.

5. Tomlins SA, Rhodes DR, Perner S, et al: Recurrent fusion of TMPRSS2 and ETS transcription factor genes in prostate cancer. Science 310: 644-648, 2005.

6. Tomlins SA, Mehra R, Rhodes DR, et al: TMPRSS2:ETV4 gene fusions define a third molecular subtype of prostate cancer. Cancer Res 66: 3396-3400, 2006.

7. Seth A and Watson DK: ETS transcription factors and their emerging roles in human cancer. Eur J Cancer 41: 2462-2478, 2005 .

8. Vaarala MH, Porvari K, Kyllonen A, Lukkarinen O and Vihko P: The TMPRSS2 gene encoding transmembrane serine protease is overexpressed in a majority of prostate cancer patients: detection of mutated TMPRSS2 form in a case of aggressive disease. Int J Cancer 94: 705-710, 2001.

9. Vaarala MH, Porvari KS, Kellokumpu S, Kyllonen AP and Vihko PT: Expression of transmembrane serine protease TMPRSS2 in mouse and human tissues. J Pathol 193: 134-140, 2001.
10. Kim TS, Heinlein C, Hackman RC and Nelson PS: Phenotypic analysis of mice lacking the Tmprss2-encoded protease. Mol Cell Biol 26: 965-975, 2006.

11. Soller MJ, Isaksson M, Elfving P, Soller W, Lundgren R and Panagopoulos I: Confirmation of the high frequency of the TMPRSS2/ERG fusion gene in prostate cancer. Genes Chromosomes Cancer 45: 717-719, 2006.

12. Åman P: Fusion oncogenes in tumor development. Semin Cancer Biol 15: 236-243, 2005.

13. Roix JJ, McQueen PG, Munson PJ, Parada LA and Misteli T: Spatial proximity of translocation-prone gene loci in human lymphomas. Nat Genet 34: 287-291, 2003.

14. Yoshimoto M, Joshua AM, Chilton-Macneill S, Bayani J, Selvarajah S, Evans AJ, Zielenska M and Squire JA: Threecolor FISH analysis of TMPRSS2/ERG fusions in prostate cancer indicates that genomic microdeletion of chromosome 21 is associated with rearrangement. Neoplasia 8: 465-469, 2006

15. Kovar H: Context matters: The hen or egg problem in Ewing's sarcoma. Semin Cancer Biol 15: 189-196, 2005.

16. Barbouti A, Höglund M, Johansson B, et al: A novel gene, MSI2, encoding a putative RNA-binding protein is recurrently rearranged at disease progression of chronic myeloid leukemia and forms a fusion gene with HOXA9 as a result of the cryptic $\mathrm{t}(7 ; 17)(\mathrm{p} 15 ; \mathrm{q} 23)$. Cancer Res 63: 1202-1206, 2003.

17. Graux C, Cools J, Melotte C, et al: Fusion of NUP214 to ABL1 on amplified episomes in T-cell acute lymphoblastic leukemia. Nat Genet 36: 1084-1089, 2004.

18. Paulsson K, Bekassy AN, Olofsson T, Mitelman F, Johansson B and Panagopoulos I: A novel and cytogenetically cryptic $t(7 ; 21)(p 22 ; q 22)$ in acute myeloid leukemia results in fusion of RUNX1 with the ubiquitin-specific protease gene USP42. Leukemia 20: 224-229, 2006.

19. Asp J, Persson F, Kost-Alimova M and Stenman G: CHCHD7PLAG1 and TCEA1-PLAG1 gene fusions resulting from cryptic, intrachromosomal 8q rearrangements in salivary gland pleomorphic adenomas. Genes Chromosomes Cancer 45: 820-828, 2006.

20. Behboudi A, Enlund F, Winnes M, et al: Molecular classification of mucoepidermoid carcinomas-Prognostic significance of the MECT1-MAML2 fusion oncogene. Genes Chromosomes Cancer 45: 470-481, 2006.

21. Lestou VS, O'Connell JX, Robichaud M, et al: Cryptic t(X;18), ins $(6 ; 18)$, and SYT-SSX2 gene fusion in a case of intraneural monophasic synovial sarcoma. Cancer Genet Cytogenet 138: 153-156, 2002.

22. Sjögren H, Meis-Kindblom JM, Örndal C, Bergh P, Ptaszynski K, Aman P, Kindblom LG and Stenman G: Studies on the molecular pathogenesis of extraskeletal myxoid chondrosarcomacytogenetic, molecular genetic, and cDNA microarray analyses. Am J Pathol 162: 781-792, 2003.

23. Mitelman F, Johansson B and Mertens F: Fusion genes and rearranged genes as a linear function of chromosome aberrations in cancer. Nat Genet 36: 331-334, 2004.

24. Petrovics G, Liu A, Shaheduzzaman S, et al: Frequent overexpression of ETS-related gene-1 (ERG1) in prostate cancer transcriptome. Oncogene 24: 3847-3852, 2005. 